\title{
Estudio cienciométrico de la actividad científica de Cuba en las Ciencias Naturales e Ingeniería y Matemática-Ciencias de la Computación
}

\author{
Darlenis Herrera Vallejera ${ }^{\star}$ \\ Rubén Sánchez Perdomo \\ Marinelsy Rosario Sierra** \\ Yaniris Rodríguez Sánchez ${ }^{\star \star \star}$
}

Artículo recibido:

9 de noviembre de 2015.

Artículo aceptado:

9 de septiembre de 2016.
RESUMEN

La cienciometría es un área disciplinar que permite evaluar la actividad científica de un país. En su haber se pueden definir políticas científicas a nivel gubernamental para determinar nuevas líneas de investigación. El objetivo de este estudio fue visualizar el desarrollo de la actividad científica cubana en las áreas del conocimiento de Matemática-Ciencias de la Computación y Ciencias Naturales e Ingeniería. En Cuba se invirtió aproximadamente el $2.7 \%$ del PIB en la actividad de

* Instituto de Información Científica y Tecnológica (IDICT), Cuba. vallejera76@gmail.com

** UNAM, Posgrado en Bibliotecología y Estudios de la Información, México. rubenperdom088@gmail.com mari.rosario9106@gmail.com

*** Cinvestav, México. yrs201181@gmail.com

INVESTIGACIÓN BIBLIOTECOLÓGICA, Vol.31, Núm.72, mayo/agosto, 2017, México, ISSN: 2448-8321. pp. 113-137 
I+D y el $63 \%$ del PIB en la educación superior según datos del Banco Mundial. Como resultado de este trabajo se determinó que las temáticas de Ciencias Químicas, Ingenierías y Astronomía-Astrofísica son las más destacadas en cuanto a producción, consumo e impacto científico. Estos resultados se debieron en gran medida a los lazos de colaboración internacional, principalmente con países como España y México. Este estudio nos indica que Cuba, que es uno de los países en vías de desarrollo de la región, prioriza buena parte de sus ingresos económicos al desarrollo de las ciencias naturales.

Palabras clave: Cienciometría; Ciencias Naturales e Ingeniería; Matemática-Ciencias de la Computación.

\section{Abstract}

Scientometrics study of scientific activity in Cuba in the fields of Natural Sciences and Engineering, Mathematics and Computer Science

Darlenis Herrera-Vallejera, Rubén Sánchez-Perdomo, Marinelsy Rosario-Sierra and Yaniris Rodríguez-Sánchez

Scientometrics provides tools for evaluating scientific activity of a nation, thereby aiding policy makers in setting policies aimed at determining new lines of research. The aim of this study is gain a clearer picture of Cuban scientific activity in the areas of Mathematics, Computer Science, Natural Sciences and Engineering. Cuba invests approximately $2.7 \%$ of GDP in $\mathrm{R} \& \mathrm{D}$ and $63 \%$ of GDP in higher education according to the World Bank. Our study shows that subject areas of Chemistry, Engineering and Astronomy-Astrophysics are the most important in terms of scientific output, consumption and scientific impact. These results are due mostly to international cooperation, mainly with Spain and Mexico. This study indicates that Cuba, a developing countries of the region, prioritizes much of its GDP to the development of natural sciences.

Keywords: Scientometry; Science and Engineering; Mathematics and Computer Science. 


\section{INTRODUCCIÓN}

$\mathrm{C}^{1}$ uso eficiente de los recursos financieros de los gobiernos dedicados al ¿quehacer científico requiere de la planeación estratégica en función de los resultados esperados. Para ello, el desarrollo de proyectos de $\mathrm{I}+\mathrm{D}+\mathrm{i}$ precisa del análisis confiable de datos sobre los recursos empleados. Debido a esto, en las modernas economías los objetivos prioritarios de la política científica de los países son el desarrollo de la ciencia, la tecnología y la innovación con base en la actividad de investigación y desarrollo (Sancho, 2003). Algunos países de América Latina, líderes en la región, implementaron iniciativas que permiten recolectar datos acerca de la actividad científico-técnica del país para determinar políticas científicas y definir líneas de investigación, tales como el Observatorio Colombiano de Ciencia y Tecnología (OCyT), el Observatorio Venezolano de Ciencia, Tecnología e Innovación (OCTI), el Observatorio Chileno de Ciencia y Tecnología (KAWAX) y el Observatorio Peruano de Ciencia, Tecnología, Sociedad e Innovación (OEI-CAEU) (De la Vega, 2009).

Estos sistemas emplean indicadores cienciométricos, entre otros, para identificar y asociar los input y output de los sistemas de ciencia, tecnología e innovación. En este sentido, y sumado al hecho de que en Cuba se avanza en la reorganización de una economía basada en el conocimiento y en nuevas formas productivas, es importante visualizar el estado de la ciencia cubana y determinar las temáticas líderes para contribuir a la planificación de los presupuestos en función de la actividad científica.

Algunos estudios cienciométricos realizados con anterioridad en Cuba incluyen el análisis de diversas temáticas, entre las que se pueden citar enfermedades infecciosas, genética humana, energía renovable, neurociencias, farmacología y nanociencias (Araujo-Ruiz et al., 2010; Soriano-Torres y Arencibia-Jorge, 2010; Collymore-Rodríguez et al., 2008; Dorta Contreras et al., 2008; Hung Llamos, Arencibia-Jorge y Araújo-Ruiz, 2008; Dayán-Aguiar et al., 2012). En estas investigaciones los autores se limitan al análisis cienciométrico a partir de indicadores bibliométricos sin establecer las relaciones con indicadores socioeconómicos, lo cual resulta insuficiente al aplicarse a la gestión de políticas científicas (Arencibia-Jorge et al., 2013; Arencibia-Jorge y Moya-Anegón, 2008).

En este sentido se evidencia la importancia de realizar investigaciones cienciométricas que respondan a cómo las políticas científicas contribuyen al desarrollo de las estructuras sociocientíficas con el soporte económico para incrementar el impacto de la ciencia cubana, y cómo estas capacidades favorecen la sostenibilidad económica. 
$\mathrm{Al}$ respecto, los estudios cienciométricos que permitan de manera integrada reflejar el estado de la producción del conocimiento científico de un país, su visibilidad e impacto, así como identificar las relaciones económicas que apoyen los procesos de investigación e innovación son de vital de importancia para lograr un mejor aprovechamiento del potencial científico y la distribución de los recursos financieros (Bornmann y Hans-Dieter, 2009; Ball, 2007).

Debido a lo antes expuesto la novedad de la presente investigación radica en contribuir al uso de las prácticas cienciométricas integradoras para la gestión de políticas científicas de un país. El aporte fundamental de esta contribución es establecer la relación entre la dimensión económica (PIB, gastos en I+D y gastos en educación) y la dimensión bibliométrica (producción, impacto y colaboración científica).

En este orden de ideas, el objetivo de este trabajo es analizar la actividad científica cubana en las áreas del conocimiento Matemática-Ciencias de la Computación y Ciencias Naturales e Ingenierías a partir de un enfoque cienciométrico integrador. Los resultados permitieron visualizar los artículos publicados y el impacto que estos generan, así como los núcleos científicos que se forman.

\section{Materiales y MÉTODOS}

La metodología bibliométrica usada se desarrolló e implementó por el Instituto de Información Científica y Tecnológica de Cuba para la evaluación de la actividad científica cubana (Rodríguez-Sánchez, 2010).

Criterios de selección de las bases de datos:

a) Paradigma tradicional para el análisis del impacto.

b) Prestigio internacional.

Fase I. Compilación de la producción científica:

a) Se estableció una estrategia de búsqueda a partir de la filiación insti tucional de Cuba limitada por el periodo de tiempo 2010-2012.

b) Se seleccionó toda la producción científica cubana procesada en el Science Citation Index, sin realizar distinción por los tipos de artí culos científicos.

c) Se empleó el EndNote X6 para la exportación de los registros bi bliográficos. 
Fase II. Normalización de la producción científica. Se normalizó a nivel de agregación meso el nombre de las instituciones, subdisciplinas y citas.

Fase III. Clasificación temática:

a) Se empleó para la clasificación temática de la producción científica la normativa del Centre for Science and Technology Studies de la Universidad de Leiden, Holanda (CWTS, 2012), tal y como se mues tra en la Tabla 1.

Tabla 1. Clasificación temática por área del conocimiento de la producción científica cubana

\begin{tabular}{|l|l|}
\hline \multicolumn{1}{|c|}{ Áreas del conocimiento } & \multicolumn{1}{c|}{ Temáticas } \\
\hline Ciencias Naturales e Ingeniería & Astronomía y Astrofísica \\
\cline { 2 - 2 } & Ciencias Nucleares \\
\cline { 2 - 2 } & Física-Matemática \\
\cline { 2 - 2 } & Nanociencias-Nanotecnología \\
\cline { 2 - 2 } & Ciencias Químicas \\
\cline { 2 - 2 } & Química-Física \\
\cline { 2 - 2 } & Ingeniería \\
\hline Matemática-Ciencias de la Computación & Matemática-Ciencias de la Computación \\
\hline
\end{tabular}

Fase IV. Aplicación de indicadores cienciométricos:

a) Indicadores bibliométricos: productividad científica; colaboración científica, consumo científico; impacto científico.

b) Indicadores macro-econonómicos: PIB, crecimiento del PIB, gasto en $\mathrm{I}+\mathrm{D}$; gastos en la educación superior.

Para el análisis de los grupos de impactos (cuartiles) se consideró el modelo Scimago Journal Rank debido a que refleja menos sesgos de impacto al considerar una ventana de tres años de citas y porque en el algoritmo de este modelo se reconoce el origen de la fuente citante.

\section{Resultados}

Con el objetivo de ofrecer de manera clara los resultados obtenidos de la dimensión bibliométrica en las áreas del conocimiento, se establecieron dos zonas: alta y baja. 
Criterios de la zona alta:

a) Alta productividad científica (superior a 200 artículos publicados al año).

b) Alto consumo científico (superior a 150 artículos citados al año).

c) Alto impacto científico (superior a 900 citas recibidas al año).

Criterios de la zona baja:

a) Baja productividad científica (inferior a 100 artículos publicados al año).

b) Bajo consumo científico (inferior a 20 artículos citados al año).

c) Bajo impacto científico (inferior a 100 citas recibidas al año).

Al realizar un análisis de la producción científica de Cuba en las áreas del conocimiento Matemática-Ciencias de la Computación y Ciencias Naturales e Ingenierías, se identificó un total de 881 artículos científicos, de los cuales 594 (67 \%) reciben 5061 citas, tal como muestra la Figura 1. A su vez, se identifica respecto al impacto científico que entre ambas áreas se obtienen como promedio seis citas por artículo publicado (impacto esperado) y siete citas por artículo citado (impacto observado), lo cual representa un puntaje de 0.5 (artículo citado por cada artículo publicado). Teniendo en cuenta este análisis se evidencia que todas las temáticas, excepto Matemática-Ciencias de la Computación e Ingeniería, presentan un puntaje superior a la media del comportamiento general. En este sentido destaca que Química-Física -la cual es la temática de menor productividad- es la única que obtiene un puntaje de la unidad ya que todos los artículos publicados reciben al menos tres citas.

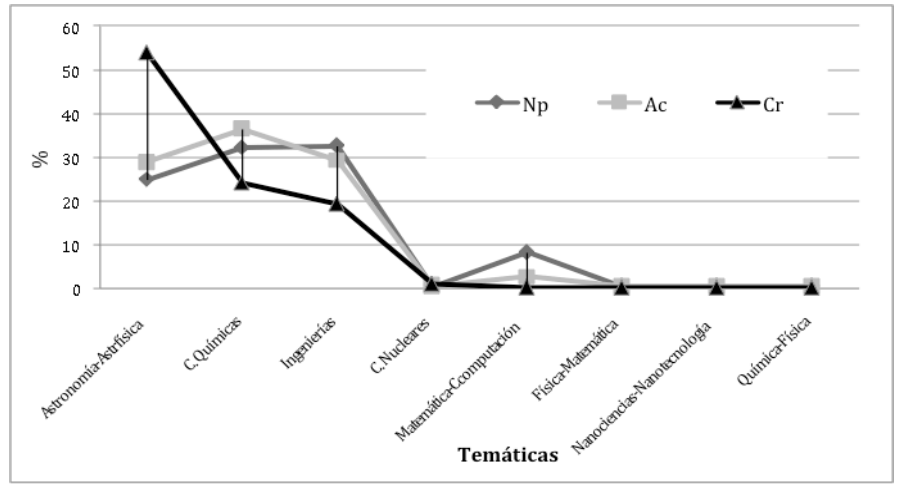

Figura 3. Comportamiento científico de las temáticas que conforman las áreas de Matemáticas- Ciencias de la Computación y Ciencias Naturales e Ingenierías 
La producción científica de las revistas corrobora que para las áreas disciplinarias del estudio y sobre la producción científica cubana existe un solapamiento al $100 \%$ a nivel de título de revistas científicas entre las bases de datos Science Citation Index y Scopus

Por su parte, el análisis del posicionamiento del impacto de las revistas científicas reflejó que el Q1 concentra la mayor producción científica (40 \%) y reúne el $51 \%$ del total de artículos citados; a pesar de esto las revistas Q4 son las que reciben el mayor impacto observado. Al respecto, los artículos procesados en Q4 recibieron como mínimo 20 citas por cada artículo citado, siendo este cuartil uno de los que atrae gran cantidad de citas (64\%) en tan pocos artículos citados, tal como se muestra en la Figura 2.

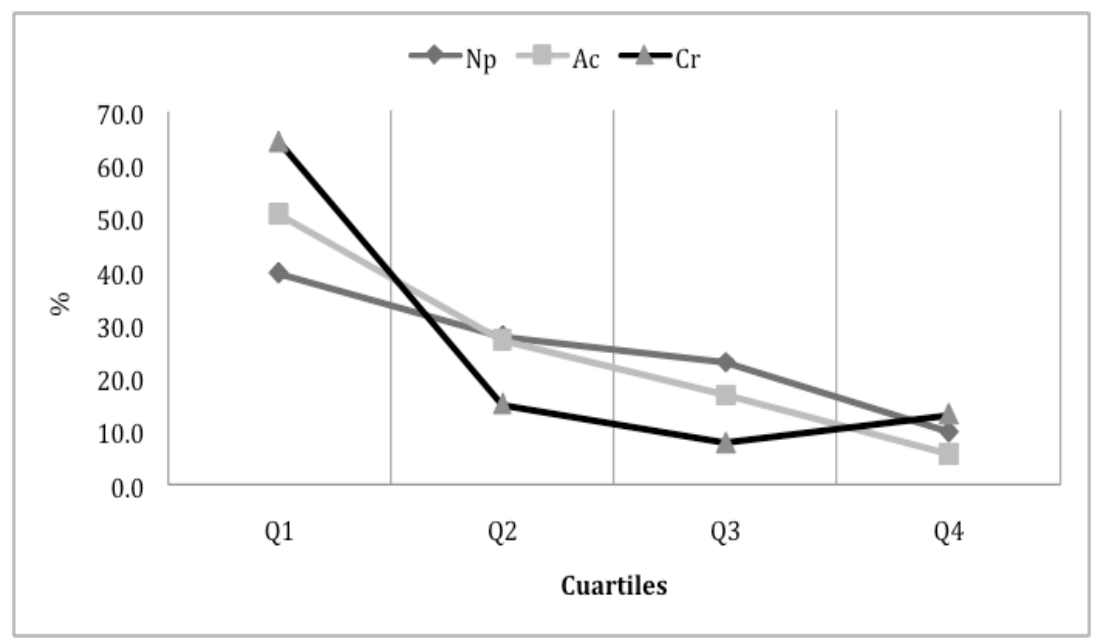

Figura 2. Comportamiento científico de los cuartiles

Nota: total de artículos publicados: Np; total de artículos citados: Ac; total de citas recibidas: Cr. Q1: cuartil 1, Q2: cuartil 2, Q3: cuartil 3 y Q4: cuartil 4.

\section{Dimensión de producción cientifica}

\section{Características de la zona alta}

La producción científica a partir de la distribución por las zonas establecidas muestra que las áreas del conocimiento Ingenierías, Ciencias Químicas y Astronomía-Astrofísica se agrupan en la zona alta debido a que representan el $90 \%$ del total de artículos publicados en el estudio. El total de artículos científicos de la zona alta es de 790, de los cuales el $41 \%$ es publicado en 
revistas Q1. El promedio de artículos publicados por esta zona es de 263 artículos por año. Un análisis puntual establece que el total de artículos publicados en Q1 es de 348, de ellos el 93 \% son artículos de la zona alta.

En este sentido se debe destacar que las temáticas de Ingeniería y Ciencias Químicas son las que logran la mayor productividad con 33 \% (287) y $32 \%$ (284) de los artículos publicados, respectivamente, en relación al total de la producción científica. Estas temáticas tienen un promedio de publicación anual de aproximadamente 96 artículos científicos. Sin embargo, a pesar de que la temática de Astronomía-Astrofísica es la de menor productividad en la zona alta, logra procesar el $31 \%$ de los artículos en revistas Q1.

\section{Características de la zona baja}

La zona baja quedó conformada por Matemática-Ciencias de la Computación, Ciencias Nucleares, Química-Física, Nanociencia-Nanotecnología y Física-Matemática, ya que representan sólo el $10 \%$ de la producción científica total del estudio. En este orden, se identifican 91 artículos publicados en la zona baja, sólo el 26 \% es publicado en revistas Q1. El comportamiento promedio de la zona es publicar 30 artículos por año.

En el caso de Matemática-Ciencias de la Computación, de los 91 artículos publicados en esta zona, 72 son resultados de investigación (79 \%), lo cual marca un rasgo distintivo en relación al resto de las temáticas de la zona baja, siendo su promedio anual de publicación de 24 artículos científicos. Sin embargo, aunque el resto de las temáticas ocupan un $21 \%$, es importante reconocer que el $63 \%$ de las mismas son procesados en revistas de Q1.

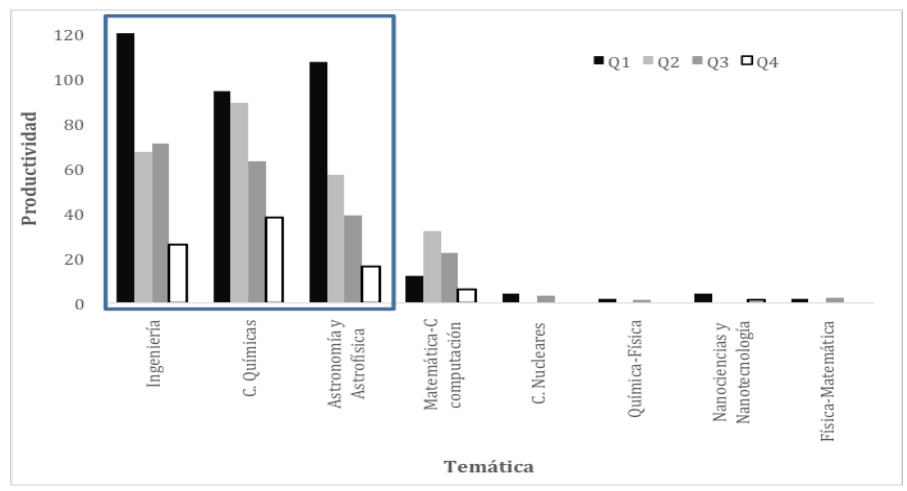

Figura 3. Producción científica en el Science Citation Index por cada cuartil en el periodo 2010-2012 Nota: total de artículos publicados 881; Q1: cuartil 1, Q2: cuartil 2, Q3: cuartil 3 y Q4: cuartil 4 
Tal y como se muestra en la Figura 3, la productividad en el cuartil 1 representa un $39.5 \%$ del total de los artículos publicados. De manera similar la distribución de la producción científica en los cuartiles 2 y 3 concentran el 27.8 \% y 22.8 \% de la producción científica, respectivamente. Este hecho demuestra una tendencia a incrementar la visibilidad y el impacto de los resultados de investigación de la comunidad científica cubana.

\section{Dimensión de consumo científico}

Teniendo en cuenta que el total de artículos citados es 594, se muestra en la Figura 4 la distribución del consumo científico (artículos citados) de las temáticas en cada cuartil.

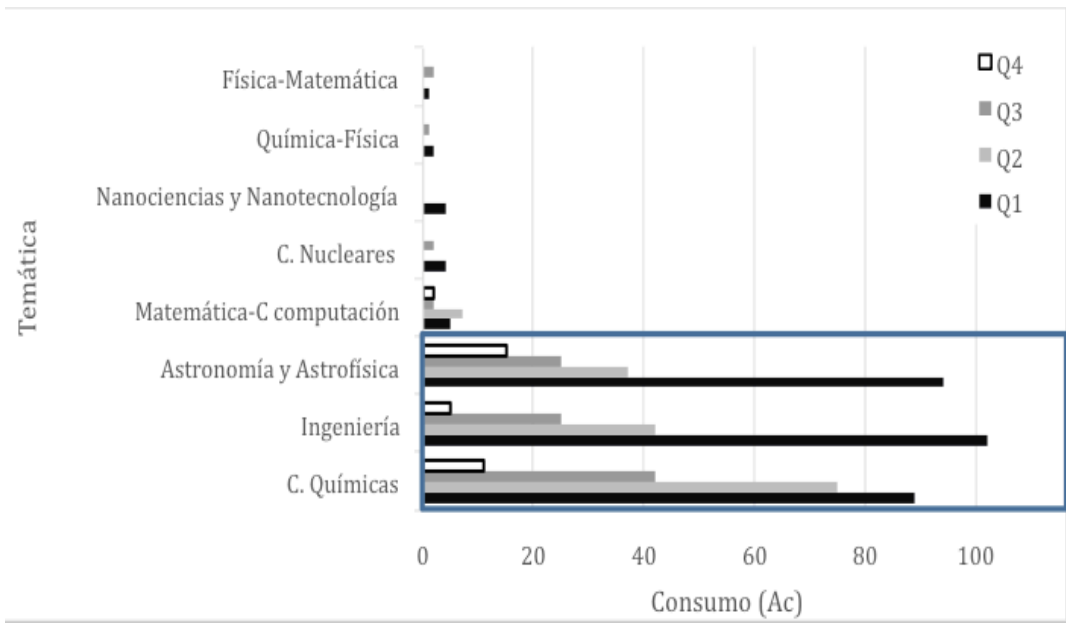

Figura 4. Distribución de artículos citados por cada temática según cuartiles en el periodo 2010-2012 Nota: total de artículos citados 594; Q1: cuartil 1, Q2: cuartil 2, Q3: cuartil 3 y Q4: cuartil 4

\section{Características de la zona alta}

Al igual que en el análisis precedente, el consumo científico distingue dos zonas de diferentes comportamientos. Las temáticas de mayor y menor consumo científico coinciden con la distribución disciplinar de la zona alta y baja de productividad científica analizada.

El total de artículos citados en la zona alta representa el $95 \%$ del total de la producción científica citada, con 562 artículos citados. La media anual de artículos citados es de 187 aproximadamente. A manera general, esta zona representa el $95 \%$ de los artículos citados Q1. No obstante, si 
bien las Ciencias Químicas sobresalen como temática de mayor consumo científico con el $37 \%$ respecto al total de artículos citados en el estudio, sólo el $41 \%$ de los mismos son artículos Q1. Las temáticas de Ingeniería y Astronomía-Astrofísica tuvieron un consumo científico de aproximadamente el $29 \%$ cada una respecto al total de contribuciones citadas del estudio, ambas se destacan por tener más del $55 \%$ de sus artículos citados procesados en el primer cuartil de impacto.

\section{Características de la zona baja}

En cuanto a la zona baja, representa el 5.4 \% de la totalidad de la producción científica citada, con 32 artículos citados. El consumo científico de esta zona tiene un promedio de 10 artículos citados por año. En este sentido se debe añadir que la zona baja representa el $5.3 \%$ respecto al total de artículos citados en Q1 (301).

Destaca el caso particular de la temática de Matemática-Ciencias de la Computación, ya que es la única en la zona baja que tiene una representación de artículos citados en todos los cuartiles. Además, se debe enfatizar que esta temática por sí sola ocupa el $50 \%$ de los artículos citados de la zona baja. No obstante, a pesar de que el resto de las temáticas suman el otro $50 \%$ de artículos citados, es significativo sobresaltar que el $69 \%$ de los mismos son procesados en revistas Q1.

\section{Dimensión de impacto científico}

Al analizar el impacto científico se obtienen 5061 citas recibidas en el estudio, las cuales se distribuyen por cada temática según cuartil de impacto (Figura 5 y Tabla 2).

\section{Características de la zona alta}

Se determinaron dos zonas de comportamiento, como ya se mencionó, y de manera coincidente las temáticas quedaron distribuidas en las mismas zonas para el caso de la variable citas recibidas.

La zona alta se distingue por ser la más destacada con 4925 citas recibidas, lo cual representa el $97.3 \%$ del total de las citas recibidas en el estudio. El comportamiento promedio anual es de alrededor de 1642 citas recibidas. En esta zona se representan el $98.4 \%$ de las citas recibidas Q1.

Al realizar una distinción por temáticas que integran la zona alta se aprecia que la que ejerce mayor influencia ante la comunidad científica es 
Astronomía-Astrofísica, con 2726 citas recibidas (53\%) de todo el estudio, de las cuales 1750 (64\%) corresponden a artículos Q1. De esta forma, constituye el núcleo citacional de la zona alta con el $55 \%$ de citas recibidas de esta zona. En este orden de ideas, al realizar una comparación en relación a las Ciencias Químicas e Ingeniería se establece que, por cada artículo citado, Astronomía-Astrofísica recibe como promedio el doble y el triple de las citas respecto a estas disciplinas científicas. De igual modo, esta temática es la que genera el mayor impacto esperado y observado de ambas zonas pues por cada artículo publicado y citado se registran 12 y 16 citas recibidas, respectivamente.

Por su parte, las Ciencias Químicas reciben la mitad de las citas totales y las Ingenierías un tercio de las citas de la zona alta. Del análisis del cuartil 1 se muestra que entre ambas poseen el $45 \%$ de las citas totales de la zona alta.

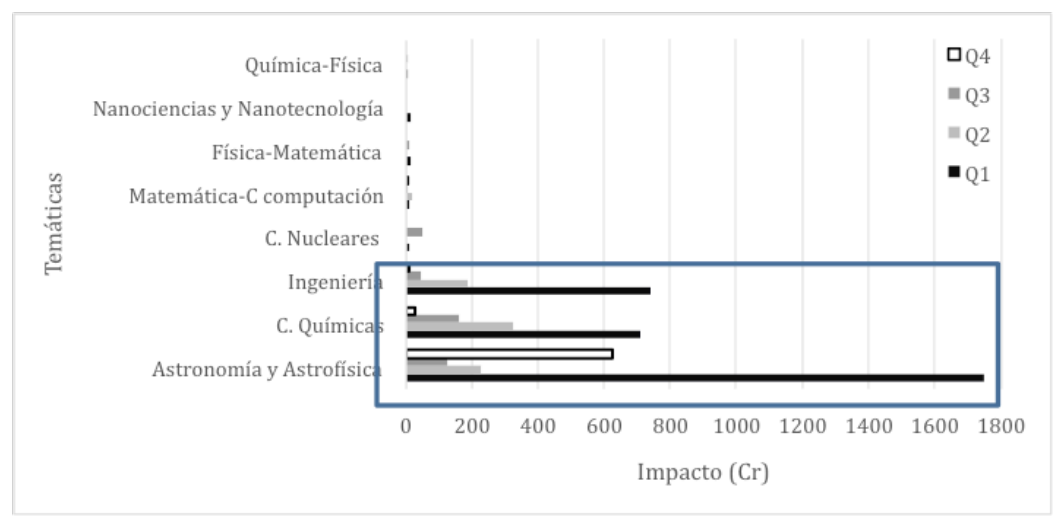

Figura 5. Distribución de las citas recibidas por temáticas según cuartil durante el periodo 2010-2012 Nota: total de citas recibidas 5 061; Q1: cuartil 1, Q2: cuartil 2, Q3: cuartil 3 y Q4: cuartil 4

\section{Caracteristicas de la zona baja}

La producción científica total de las temáticas de la zona baja recibió 136 citas $(2.7 \%)$. La media anual de citas recibida es aproximadamente 45 . Se debe destacar que el $38.2 \%$ de las citas recibidas en la zona baja corresponden a artículos Q1.

Resaltan las disciplinas de las Ciencias Nucleares y la Física-Matemática, en ambas áreas se observan comportamientos de impacto esperado e impacto observado con valores superiores a dos temáticas (Ciencias Químicas e Ingenierías) de las tres que conforman la zona alta. 
En este sentido, Ciencias Nucleares sigue un patrón de impacto peculiar, pues el $83 \%$ de los artículos citados de esta disciplina están en revistas Q3, sin embargo, logra atraer ocho citas por cada artículo publicado y 10 citas por cada artículo citado, representando el núcleo citacional de la zona baja con 58 citas recibidas (43 \%). En este desempeño, la temática de Ciencias Nucleares supera en 24 citas recibidas, en cinco veces al impacto observado y en ocho veces al impacto esperado con respecto a la temática Matemática-Ciencias de la Computación, que es la de mayor productividad científica de la zona baja.

Tabla 2. Distribución de las citas recibidas por temáticas según cuartil durante el periodo 2010-2012

\begin{tabular}{|l|c|c|c|c|c|}
\hline \multicolumn{1}{|c|}{ Temática } & Np & Ac & Cr & Cr/Ac & Cr/Np \\
\hline Astronomía y Astrofísica & 219 & 171 & 2726 & 16 & 12 \\
\hline C. Químicas & 284 & 217 & 1220 & 6 & 4 \\
\hline Ingeniería & 287 & 174 & 979 & 6 & 3 \\
\hline C. Nucleares & 7 & 6 & 58 & 10 & 8 \\
\hline Matemática-C. Computación* & 72 & 16 & 34 & 2 & 0 \\
\hline Física-Matemática & 4 & 3 & 22 & 7 & 6 \\
\hline Nanociencias-Nanotecnología & 5 & 4 & 13 & 3 & 3 \\
\hline Química-Física & 3 & 3 & 9 & 3 & 3 \\
\hline TOTAL & 881 & 594 & 5061 & 9 & 6 \\
\hline $\begin{array}{l}\text { Nota: sombreado se representa la zona alta, donde total de artículos citados (Ac) 594, total de artículos } \\
\text { publicados (Np) 881, total de citas recibidas (Cr) 5 061 en negritas los valores por encima de la media. }\end{array}$ \\
*Esta área del conocimiento recibe matemáticamente 0.5 citas por artículos publicados. \\
\hline
\end{tabular}

El análisis de ambas zonas demuestra comportamientos muy diferentes entre la zona baja y alta no sólo para el caso de la dimensión de producción científica, sino también para el caso de las citas recibidas, donde en su conjunto la zona alta supera en impacto 36 veces más que la zona baja.

Por su parte, la zona alta muestra un desempeño disciplinar bien definido por un área del conocimiento consolidada, donde la Astronomía-Astrofísica es el núcleo de la producción científica que sobresale en visibilidad y en impacto.

Sin embargo, en el comportamiento de las cinco disciplinas científicas de la zona baja no se distinguen patrones homogéneos de visibilidad e impacto. Se destaca las Ciencias Nucleares y la Física-Matemática por el nivel de impacto de artículos Q1 y Q3, mientras que son del área de la Matemática-Ciencias de la Computación los artículos Q2 que reciben la mayoría de las citas de este campo científico; para el caso de las Nanociencias y Nanotecnología sólo los artículos Q1 publicados de esta temática son los que reciben citas. 


\section{Colaboración cientifica}

En la Figura 6 se visualizó una red de colaboración en la que los resultados representan a aquellas instituciones con más de dos artículos en colaboración en las temáticas de Matemática-Ciencias de la Computación.

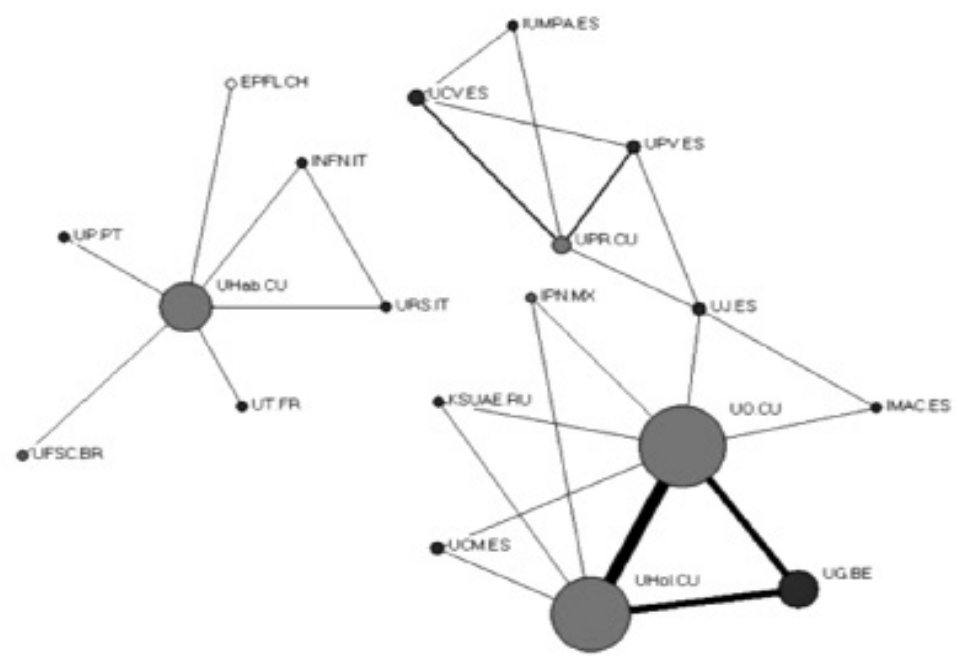

Figura 6. Coloboración internacional de las instituciones en la temática de matemática-ciencia de la computación

Leyenda:

- Instituciones de la Comunidad Europea

Instituciones cubanas

Instituciones de Asia

Instituciones de América

En la red de colaboración hay un $31.6 \%$ de instituciones cubanas. La Universidad de Oriente (UO.CU) es la que establece mayor colaboración (19 instituciones) de la cuales el 47 \% son colaboración nacional. En el caso de la colaboración internacional de la misma predominan los trabajos con Europa (32\%), le sigue América con un $16 \%$ y Asia sólo representa un $5 \%$ de la colaboración con otros países.

Al realizar el análisis de la red con respecto al grado de centralidad y de intermediación se destaca la Universidad de Oriente. Esta universidad obtiene el máximo valor en ambos parámetros (48.000 y 33.000, respectivamente) ratificándose como la institución que más colabora y la que sirve como puente 
entre diferentes entidades. En el caso del análisis de centralidad ocupa un segundo lugar la Universidad de Holguín, pero en el análisis de intermediación es la Universidad de La Habana quien es segunda en la lista de las entidades que actúan como puente entre diferentes entidades.

En la Figura 7 se observa una red de más de cuatro artículos en colaboración en las temáticas de Ciencias Químicas, Nanociencias-Nanotecnología y Química-Física.

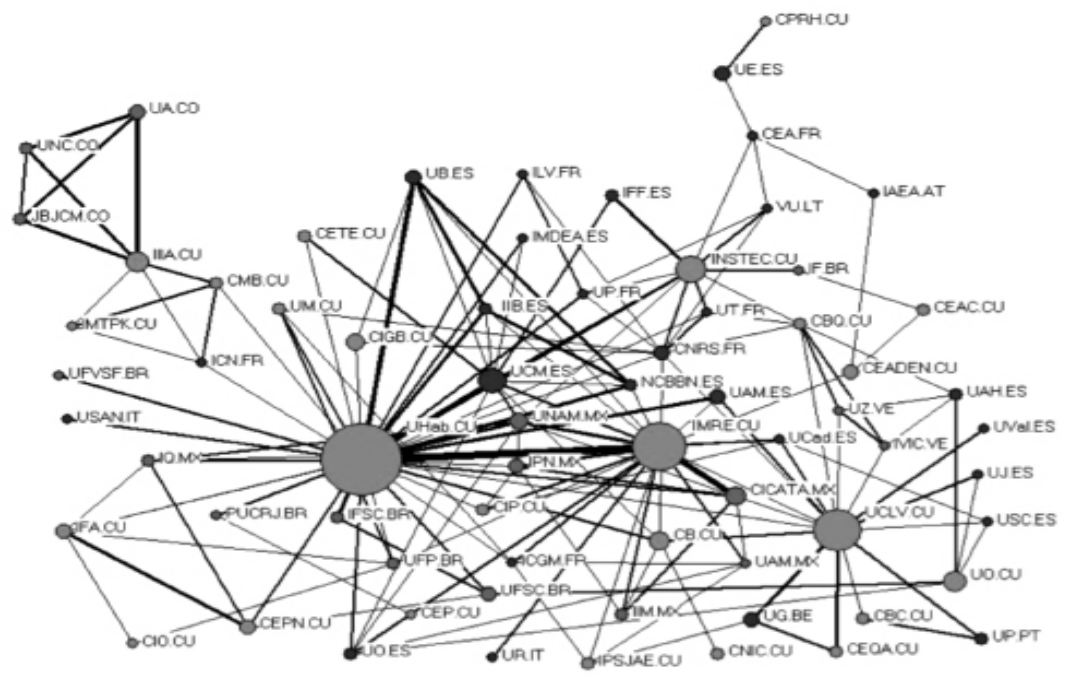

Figura 7. Colaboración internacional de las instituciones cubanas en la temática de Ciencias Químicas, Nanociencias-Nanotecnología y Química-Física

Leyenda:

- Instituciones de la Comunidad Europea

- Instituciones cubanas

Instituciones de América

La red se muestra simétrica donde las instituciones cubanas representan un $23.4 \%$. La Universidad de La Habana (UHab.CU) es la institución de mayor colaboración de la red (109 instituciones), donde la colaboración nacional ocupa el $19 \%$. En la colaboración internacional de esta universidad predominan los países del continente latinoamericano (35\%), mientras que el $19 \%$ de las contribuciones científicas se realizaron de conjunto con entidades de Europa. 
En relación con el grado de centralidad y al grado de intermediación, los resultados mostraron que la UHab.CU tiene el mayor valor en ambos parámetros (99.000 y 1274.767 , respectivamente) destacándose como la institución que más colabora y la que sirve como gestora entre diferentes entidades. Como resultado sobresaliente las redes sociocientíficas establecidas por el Instituto de Ciencia y Tecnología de los Materiales (IMRE.CU) y la Universidad Central de las Villas (UCLV.CU) ocupan un segundo lugar en cuanto a grado de centralidad y en el grado de intermediación, respectivamente.

En la Figura 8 se obtuvo una red en la que se realizó una poda a partir de cinco artículos científicos en las temáticas de Ciencias Nucleares, Astronomía-Astrofísica y Física-Matemática.

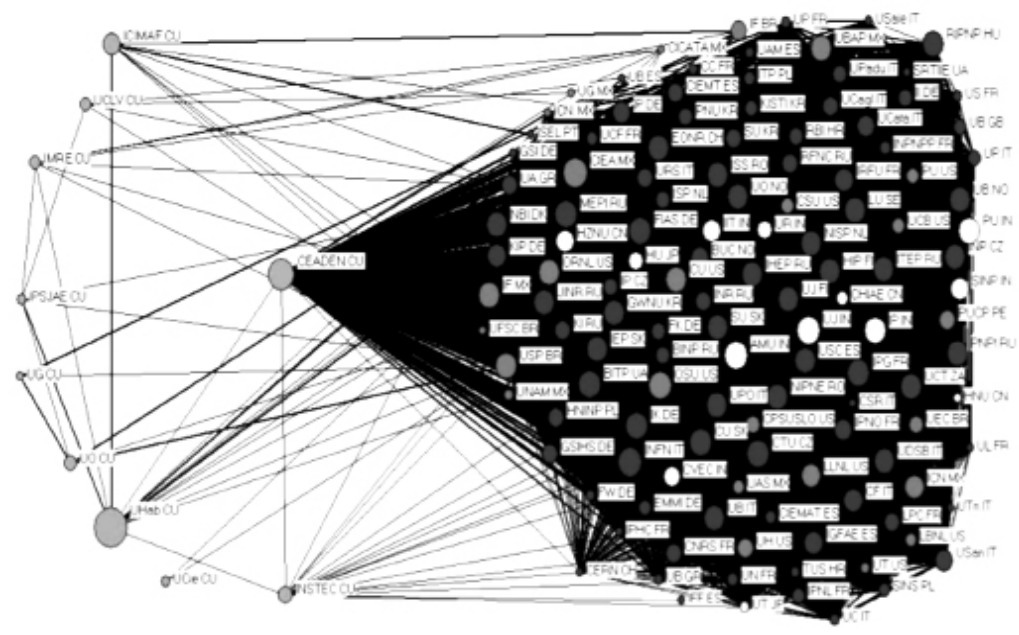

Figura 8. Colaboración internacional de las instituciones cubanas en la temática de Ciencias Nucleares Astronomía-Astrofísica y Física-Matemática

Leyenda:

Instituciones de la Comunidad Europea

Instituciones cubanas

Instituciones de América

Instituciones de Asia

Instituciones de África 
La red que se forma no es homogénea y Cuba sólo representa el 7 \%. A pesar de que las instituciones cubanas no forman parte del núcleo de la red, todas las instituciones participantes mantienen trabajos en colaboración con entidades cubanas. En la red predominan las instituciones europeas con el $56.6 \%$, le sigue América Latina en un $32 \%$, Asia con un $11.3 \%$ y el continente africano está representado con el $0.23 \%$.

La fuerte colaboración fue sobresaliente para el caso de la temática de Astronomía-Astrofísica, donde participan más de 150 instituciones que se incluyen en el proyecto de colaboración internacional ALICE (European Organization for Nuclear Research, 2012). La única institución cubana que participa en este proyecto es el Centro de Aplicaciones Tecnológicas y Desarrollo Nuclear (CEADEN.CU), siendo la de mayor grado de centralidad (3029.000) e intermediación (314.942) respecto al total de las instituciones cubanas identificadas en el estudio. En otro sentido destaca que existen instituciones cubanas que conectan una gran cantidad de entidades en el estudio, entre ellas se encuentran el Instituto Superior de Tecnologías y Ciencias Aplicadas (INSTEC.CU), la Universidad de La Habana (UHab.CU) y el Instituto de Cibernética Matemática y Física (ICIMAF.CU); las cuales tienen valores cercanos al grado de intermediación del CEADEN.CU.

En la Figura 9 se observa una red con una poda a partir de dos artículos en colaboración en las temáticas de Ingeniería.

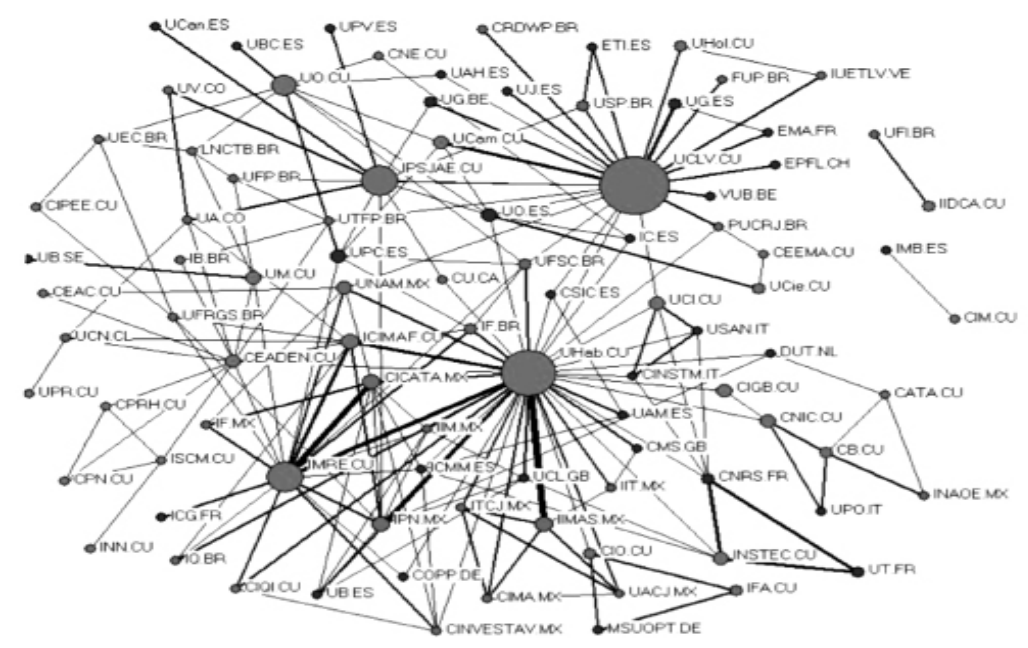

Figura 9. Relaciones de colaboración científica entre las entidades cubanas y extranjeras en el campo de la ingeniería en el Science Citation Index durante el periodo 2010-2012 
Leyenda:

- Instituciones de la Comunidad Europea

Instituciones cubanas

Instituciones de América

La red muestra que la mayoría de sus nodos tienen múltiples relaciones entre ellos, lo que demuestra la alta colaboración científica de esta temática. Las instituciones cubanas representan el $29.4 \%$ en la red, donde la Universidad de La Habana (UHab.CU) y la Universidad Central de las Villas (UCLV.CU) son las entidades que establecen la mayor colaboración en la red. La UHab.CU tienen la mayor red con 75 entidades, el $17 \%$ pertenece a la colaboración nacional. Por su parte, se identificó que los resultados publicados con investigadores internacionales corresponden al $41 \%$ y $39 \%$ de instituciones europeas y americanas, respectivamente, mientras que el $3 \%$ son de entidades asiáticas. Un comportamiento diferente se muestra en la UCLV. $\mathrm{CU}$, donde hay un mayor porcentaje de la colaboración nacional (26\%) en una red de 58 instituciones.

En el caso de la colaboración internacional predominan los trabajos en colaboración con América Latina (46 \%) por encima de un 27 \% con Europa. Al realizar un análisis de la red en cuanto al grado de centralidad y al grado de intermediación se ratifica que son estas dos instituciones las que más se destacan en el estudio: la Universidad de La Habana obtuvo 74.000 y 2174.439 seguida de la Universidad Central de Las Villas con 47.000 y 1192.990, respectivamente.

\section{Discusión}

Analizar el comportamiento de la actividad científica de Cuba entre el 2010 y el 2012 a partir del Science Citation Index en las Ciencias Naturales y las Ciencias Técnicas permitió caracterizar el comportamiento del desempeño investigativo de estas áreas del quehacer científico.

Como aspectos generales de estas áreas del conocimiento se aprecia que el $68 \%$ de las contribuciones científicas del periodo examinado corresponden a artículos Q1 y Q2, mientras que el $10 \%$ fueron artículos Q4. En este último caso, sólo la variable de impacto científico mostró un comportamiento distintivo debido a que supera los valores de las citas recibidas por artículos citados de los cuartiles 2 y 3 . Esta característica resultó de interés, ya que uno de los aspectos que se asocia al impacto de la producción científica es el factor de impacto de la revista en la cual se publican los resultados de investigación. Sin 
embargo, en esta investigación se estableció que son los artículos Q1 y Q4 los que reflejan mayor impacto.

Este comportamiento se debe a la publicación de 16 artículos científicos en una misma revista Q4 de la temática de Astronomía-Astrofísica, los cuales fueron firmados por los autores más citados de toda la muestra analizada. Estos artículos forman parte del resultado de un proyecto de colaboración internacional (ALICE) que agrupa alrededor de 200 autores que generalmente publican sus resultados de investigación en Q1. Al respecto, se cumple entonces una condición autoral del efecto halo como factor determinante para incrementar el impacto de las investigaciones científicas.

En este sentido sería importante fortalecer la visibilidad de las revistas científicas regionales a partir de programas de promoción de la comunicación científica para incentivar a investigadores líderes a que publiquen en revistas nacionales, así como incidir en las políticas de evaluación de la ciencia de la región que consideren criterios endógenos para diagnosticar el estado de las investigaciones científicas nacionales.

Al realizar un análisis integral bibliométrico de las variables de producción e impacto, son las disciplinas de la Astronomía-Astrofísica, Ciencias Químicas, Ingenierías y Ciencias Nucleares las que logran establecer un mejor efecto halo entre cantidad de artículos publicados y citas recibidas.

En este orden de ideas, la colaboración como dimensión bibliométrica determinó que los hábitos de productividad y de impacto de las disciplinas Astronomía-Astrofísica, Física-Matemática y Ciencias Nucleares están condicionados por el liderazgo socio-científico de tres entidades cubanas: Universidad de La Habana, Instituto de Matemática y Física y Centro de Aplicaciones Tecnológicas y Desarrollo Nuclear. Sobresale la baja colaboración nacional y fuerte colaboración internacional con países de casi todos los continentes, siendo esto una característica distintiva para Cuba reflejada a partir exclusivamente de estas temáticas. Ello se debe principalmente al proyecto ALICE y al hecho de que las investigaciones científicas para el desarrollo de la energía nuclear son muy costosas, por lo que la colaboración internacional ha sido una de las vías para potenciar el conocimiento científico cubano en esta disciplina.

Sin embargo, resalta que las investigaciones científicas cubanas de estas áreas del conocimiento han tenido para el país una aplicación especial en el campo de la biomedicina. Los programas de salud en Cuba constituyen una prioridad y son accesibles sin costo para todos los ciudadanos cubanos; se brindan tratamientos para la diabetes, se realizan trasplantes de células madre y se producen vacunas terapéuticas para el cáncer de pulmón, entre otras acciones en función de la salud. 
En este esfuerzo el gobierno garantiza la inmunización de los infantes con la aplicación de 12 vacunas producidas en Cuba a partir del financiamiento de las investigaciones de la industria biofarmacéutica cubana, algunas de ellas como la Haemophilus Influenzae (Hib) con una patente exclusiva de Cuba. El costo de adquisición de esta vacuna es siete veces superior al costo total de las vacunas contra el sarampión, el polio, la difteria y la tos ferina (estas enfermedades fueron erradicadas en Cuba). Otro ejemplo de las características distintivas del sistema de salud cubano y que requiere de elevados costos y potencial científico altamente calificado es mantener por ocho años una tasa de mortalidad infantil por debajo de cinco por cada mil nacidos vivos, comportamiento de países como Canadá, Francia, Reino Unido, e inferior respecto a países altamente industrializados o que constituyen economías emergentes, tales como Estados Unidos, China y Federación Rusa (WHO, 2012).

En el caso de Matemática-Ciencia de la Computación se establece que, aunque su producción científica sobresale como núcleo de la zona baja, es una temática con baja colaboración liderada por la Universidad de La Habana, la Universidad de Oriente y la Universidad de Holguín. Al respecto es importante argumentar que la inserción de la Universidad de Oriente y la Universidad de Holguín al Consejo Interuniversitario Flamenco de Bélgica (VLIR) permite el fortalecimiento del intercambio académico para la formación de másteres y doctores bajo enfoques disciplinarios altamente competitivos, así como el mejoramiento de las instalaciones y laboratorios e incrementa la eficacia de la gestión integrada del sistema de universidades cubanas.

Sin embargo, el desarrollo de las investigaciones de esta área del conocimiento aún se considera en emergencia. En este particular, el gobierno cubano fundó en el 2002 la Universidad de Ciencias Informáticas (UCI), con una inversión cuantiosa para crear una Ciudad Digital Avanzada con capacidad para 20000 personas, con el objetivo de acometer acciones en ámbitos como el desarrollo de la industria de software, la cooperación internacional, las transformaciones de procesos en las entidades para asumir su informatización y el soporte necesario para su mantenimiento (UCI, 2002). Dado esto, la UCI responde a un modelo especial de desarrollo de investigaciones en el que, si bien el gobierno cubano subvenciona los gastos asociados al desarrollo del capital humano y al mantenimiento de las inmuebles de la UCI, la publicación de resultados en revistas científicas internacionales de impacto es muy bajo. En este orden se precisa revaluar por la comunidad científica de la UCI las oportunidades que pueden obtener si se insertan en los ciclos productivos de investigación, desarrollo, innovación y comercialización. 
Como es conocido, en contextos internacionales las universidades brindan servicios científicos y técnicos de interés para la industria; mediante esta relación se activa un engranaje de autogestión económica que incluye la colaboración internacional y que permite a las universidades obtener parte del financiamiento de la investigación científica.

En este orden de ideas, conviene apuntar los hábitos de colaboración científica de las temáticas de la Ciencias Químicas, Nanociencias-Nanotecnología, Química-Física y las Ingenierías, donde las universidades son las que guían y lideran la producción científica altamente de impacto. Tanto para el caso de la Universidad de La Habana como de la Universidad Central de las Villas, los modelos de la actividad científica de manera tradicional son el reflejo de proyectos de investigación internacionales que han permitido importantes resultados en las investigaciones en línea, como las ciencias de los materiales y las energías renovables.

Es importante señalar que en la relación entre impacto y colaboración interviene un factor de capacidad socio-científica disciplinar que determina a posteriori la influencia que ejercen los resultados de investigación ante la comunidad científica. Tal es el caso de este estudio, donde se demuestra que la colaboración nacional respecto a la colaboración internacional tiene un menor impacto. Este comportamiento es esperado si se considera que los enfoques disciplinarios de diferentes contextos, específicamente con participación internacional, le confieren al artículo científico mayor grado de madurez y, por ende, mayor atractivo para los investigadores a ser usados como soporte a otras investigaciones.

Un análisis cienciométrico y basado en datos del Banco Mundial demostró que Cuba es un país de ingreso mediano alto, con un PIB promedio para el periodo 2010-2012 de alrededor de 57 mil millones de dólares (a precio constante). En el periodo de análisis alcanzó un crecimiento alrededor del $2.8 \%$ del PIB (a precio constante) (Banco Mundial, 2012a). Con este lento crecimiento Cuba ocupó el sexto lugar en el Scimago Country Rankings teniendo en cuenta su productividad (Grupo SCimago, 2012a), esfuerzo que debe destacarse teniendo en cuenta que es un país no desarrollado, con programas de asistencia social y de educación altamente costosos, y con la totalidad de los servicios accesibles a todos los ciudadanos de manera gratuita.

$\mathrm{Al}$ realizar un análisis sobre gastos de I+D se identificó que Cuba empleó para la actividad de investigación y desarrollo aproximadamente $0.43 \%$ del PIB, situando al país en una posición muy cercana a otros de la región, tales como Uruguay (0.42 \%), Costa Rica (0.47 \%), México (0.45\%) y Chile (0.36 \%) (Banco Mundial, 2012b). Estos dos últimos se ubican, respectivamente, en la segunda y cuarta posición del ranking por países en el Scimago 
Country Rankings debido a su alta productividad (Grupo SCimago, 2012a).

Este gasto permitió una inversión de 876.3 millones de pesos por concepto de actividad de investigación y desarrollo en este periodo. En cuanto a adquisición de tecnología se invirtieron 71.4 millones de pesos. Para construcción y montaje de instituciones especializadas, 47.9 de millones de pesos. El gobierno, por su parte, subvencionó 1143 millones y las empresas aportan 200 millones de su financiamiento, así como 78.3 millones proveniente de otras fuentes de financiamiento, tales como proyectos internacionales u organizaciones no gubernamentales (ONG) (ONEI, 2012). Estos resultados evidencian el interés del gobierno cubano por marcar un avance en el desarrollo de las ciencias. En esta investigación se identificó que la actividad de I+D emplea millones de pesos cubanos en desarrollo de infraestructura, así como en la adquisición de tecnología altamente especializada necesaria para lograr resultados científicos de alta visibilidad.

En este sentido, Cuba tiene un sistema riguroso de selección para el ingreso al nivel superior de educación. Este sistema es coherente con las necesidades económicas y sociales del país. Las estadísticas del Banco Mundial indican que Cuba es uno de los países a nivel mundial que más invierte en educación superior (63\% del PIB per cápita). Estas estadísticas superan a las obtenidas por países como Colombia (25.5\%), Chile (15.2\%), Brasil (28.6 \%) y Argentina (15\%) (Banco Mundial, 2010). Los dos últimos ocupan las posiciones primera y tercera, respectivamente, del ranking por países en el Scimago Country Rankings debido a su alta productividad (Grupo SCimago, 2012a).

Realizando un análisis general, se estableció que Cuba tuvo en el 2012 alrededor de 11 millones de habitantes y cuenta con 63 universidades, lo que refleja un estimado de 174 mil habitantes por universidad. En este orden de ideas, se valoró que dos de estas universidades, la Universidad de La Habana y la Universidad Central de las Villas, tienen una posición dentro de la región de 79 y 177, respectivamente, en el Scimago Institutions Rankings (Grupo SCimago, 2012b).

$\mathrm{Al}$ realizar un análisis del egreso de los estudiantes del nivel superior durante el periodo de análisis se comprobó que las facultades de ingenierías del Instituto Superior Politécnico José Antonio Echevarría (IPSJAE.CU) y las facultades de Ciencias Naturales de las diferentes universidades del país tuvieron 17108 y 1752 estudiantes, respectivamente. Al respecto se identifica que 2430136 estudiantes han continuado la educación postgraduada hasta la fecha correspondiente al periodo de análisis, de los cuales 329555 (13.6\%) han cursado maestría y 15904 (0.7 \%) han realizado doctorados (ONEI, 2012). Este resultado evidenció que el gobierno cubano tiene la voluntad de 
mantener una matrícula constante de estudiantes a pesar de las condiciones económicas del país. En este estudio se confirmó que las carreras técnicas, de química y de física han formado profesionales capaces de lograr una alta producción científica en las temáticas de Ingeniería, Ciencias Químicas y Astronomía-Astrofísica.

En Cuba, como parte de la política científica implementada por el Ministerio de Ciencia, Tecnología y Medio Ambiente (CITMA), existen categorías de tres niveles. Para obtener alguna de las categorías se requiere de varios indicadores, entre los cuales se encuentra la producción científica de cada investigador. De la misma forma, para la obtención de los grados académicos es necesario reunir una serie de requisitos, entre ellos la publicación de artículos científicos en revistas indizadas en el Web of Knowledge y Scopus (CITMA, 1988). En relación a ello, Cuba tenía 219419 profesionales en la actividad científica-técnica afiliados a entidades de investigación, desarrollo e innovación durante el periodo analizado, de ellos 14145 investigadores se categorizaron científicamente (6.4\%) y 36176 obtuvieron grados científicos $(16.4 \%)(O N E I, 2012)$. A pesar de ser un núcleo pequeño de personal dedicado a la actividad de I+D ha logrado altos niveles de consumo (Ac) e impacto $(\mathrm{Cr})$ en estas temáticas, capaces de ejercer una influencia científica notable en áreas del conocimiento que tienen un ritmo de crecimiento más lento que las ciencias aplicadas (Abramo, Cicerob y D'Angelo, 2011).

En la actualidad, las políticas científicas cubanas coinciden en la implementación de ciclos productivos de investigación, desarrollo, innovación y comercialización para que el país incremente la producción científica y su impacto como garante de calidad y fiabilidad para la innovación y con ello aumentar las exportaciones al mercado internacional.

Sin embargo, la cultura basada en el conocimiento científico para el caso de Cuba es un macro-proceso complejo. Esto implica desarrollo de habilidades en la comunicación científica, gestión de la información científica, cambios en la organización y administración de las empresas y la academia para reducir la brecha universidad-industria, mantenimiento de programas de capacitación de calidad y gestión de políticas económicas que liberen al gobierno de la subvención casi total de la ciencia y la innovación. Además, se deben garantizar los compromisos sociales mantenidos históricamente desde 1959.

Desde la perspectiva integradora de la cienciométrica cabe destacar las características distintivas de Cuba respecto al desempeño de la actividad científica:

- La implementación de políticas científicas ha estado orientada a fortalecer las capacidades científicas a partir de la formación de profesionales desde la educación superior y posgraduada. 
- La productividad científica de Cuba es resultado de un sistema de educación competitivo gratuito y con oportunidades para todos.

- En el impacto de la actividad científica cubana los vínculos internacionales son un componente determinante.

\section{Conclusiones}

Cuba es un país que prioriza el fortalecimiento de programas sociales altamente costosos, donde algunos de los indicadores de salud son similares a los de países altamente industrializados como Francia, Canadá y Reino Unido; sin embargo, el nivel de industrialización en Cuba dista mucho de estos países. En este devenir, el desarrollo económico cubano desde la ciencia y la innovación aún es insuficiente, a excepción del caso de éxito de la industria biofarmacéutica.

Como principal fortaleza de Cuba, que constituye además uno de los pilares gracias al cual la colaboración científica se manifiesta como vía constante de apoyo a la investigación científica, se encuentra la implementación de programas de formación posgraduada de calidad. No obstante, la colaboración científica nacional muestra que pocas instituciones cubanas logran darle visibilidad a sus investigaciones y dependen en gran medida de la colaboración internacional, en la que predominan los lazos con países europeos (principalmente España, por la idiosincrasia y los lazos históricos que comparten) y países latinoamericanos (en particular México, por la cercanía geográfica y la tradición que une a ambos pueblos).

Las políticas científicas de Cuba siguen pautas comunes independientemente del área disciplinar en las que se implementen porque son coordinadas para responder a intereses políticos y sociales; aunque ello constituye una fortaleza para la sociedad cubana, es importante realizar evaluaciones y diseñar directrices según las características y la dinámica disciplinar de cada área del conocimiento. Ejemplo de ello es el caso de Matemática-Ciencias de la Computación, donde se observa poca colaboración y debilidad en la formación de grupos de investigadores nacionales, especialmente entre la capital y el oriente cubano, debido a lo cual se deben promover las alianzas interinstitucionales y la transferencia de conocimientos.

Aunque aún la relación de autogestión económica no es una fortaleza para el Sistema de Ciencia, Tecnología e Innovación en Cuba, en algunas áreas disciplinarias como las Ciencias Naturales y las Ingenierías los resultados de investigaciones científicas están mejor condicionadas para el desarrollo de servicios científicos y técnicos como soporte económico. 


\section{REFERENCIAS}

Abramo, Giovanni, Tindaro Cicerob y Ciriaco Andrea D’Angelo. 2011. "Assessing the varying level of impact measurement accuracy as a function of the citation window length." Journal of Informetrics 5(4): 659-667 DOI: 10.1016/j. joi.2011.06.004.

Araujo-Ruiz, Juan Antonio, Ricardo Arencibia-Jorge, Rosa Lidia Vega-Almedia y Nancy Sánchez-Tarragó. 2010. "Producción científica cubana sobre Dengue en el contexto internacional (Scopus, 1981-2006).” Revista CNIC. Ciencias Biológicas 41 (4): 1-11. https://www.researchgate.net/publication/237027091

Arencibia-Jorge, Ricardo y Félix de Moya-Anegón. 2008. "La evaluación de la investigación científica: una aproximación teórica desde la Cienciometría.” ACIMED 17 (4): 1-27. http://scielo.sld.cu/pdf/aci/v17n4/aci04408.pdf

Arencibia-Jorge, Ricardo, Elena Corera-Álvarez, Zaida Chinchilla-Rodríguez y Félix de Moya-Anegón. 2013. "Relaciones intersectoriales, producción científica y políticas nacionales para el desarrollo de la investigación: un estudio de caso sobre Cuba 2003-2007." Revista Cubana de Información en Ciencias de la Salud 24 (3): 243-254. http://scielo.sld.cu/pdf/ics/v24n3/rci03313.pdf

Ball, Philip. 2007. "Achievement index climbs the ranks.” Nature 448 (7155): 737. http://dx.doi.org/10.1038/448737a

Banco Mundial. 2010. Gasto por alumno, nivel terciario (\% del PIB per cápita). http:// datos.bancomundial.org/indicador/SE.XPD.TERT.PC.ZS

Banco Mundial. 2012a. Crecimiento del PIB (\% anual). http://datos.bancomundial. org/pais/cuba

Banco Mundial. 2012b. Gasto en investigación y desarrollo (\% del PIB). http://datos. bancomundial.org/indicador/GB.XPD.RSDV.GD.ZS/countries?display=default

Bornmann, Lutz y Daniel Hans-Dieter. 2009. "The state of h index research: is the h index the ideal way to measure research performance?” European Molecular Biology Organization 10 (1): 2-6. https://www.ncbi.nlm.nih.gov/pmc/articles/ PMC2613214/pdf/embor2008233.pdf

CITMA (Ministerio de Ciencia Tecnología y Medio Ambiente). 1988. Decreto ley sobre Otorgamiento y Pérdida de las Categorias Cientificas. http://instituciones.sld. cu/cirah/files/2013/03/08-Decreto-Ley-104-y-106-sobre-las-categor\% -

C3\%ADas-cient\%C3\%ADficas.pdf

Collymore-Rodríguez, Andrea, Ricardo Arencibia-Jorge, Armando Blanco-García y Juan Antonio Araújo-Ruiz. 2008. "Producción científica mundial sobre biodiesel." ACIMED 18 (5): 1-19. http://scielo.sld.cu/pdf/aci/v18n5/aci041108.pdf

CWTS (Centre for Science and Technology Studies). 2012. "Leiden University Ranking." Leiden University. http://www.leidenranking.com/information/fields

Dayán-Aguiar, Jorge, Ricardo Arencibia-Jorge, Juan Antonio Araujo-Ruiz y Deysi Alba-Labaut. 2012. "Producción Científica Cubana sobre Nanociencias y Nanotecnología." Ciencias de la Información, 43 (1): 5-14.

De la Vega, Iván. 2009. Banco Interamericano de desarrollo. WORKING PAPER 6 Módulo de capacitación para la recolección y el análisis de indicadores de investigación y desarrollo. http://docs.politicascti.net/documents/Doc\%2006\%20-\%20 capacitacion $\% 20 \mathrm{de} \% 201 \mathrm{a} \% 20 \mathrm{vega} . \mathrm{pdf}$ 
Dorta-Contreras, Alberto Juan, Ricardo Arencibia-Jorge, Yohannis Martí-Lahera y Juan Antonio Araújo-Ruiz. 2008. "Indicadores basados en análisis de citas para la caracterización de las neurociencias cubanas.” ACIMED 18 (6): 1-21. http:// www.bvs.sld.cu/revistas/aci/vol18_6_08/aci051208.htm

European Organization for Nuclear Research. 2012. "A Large Ion Collider Experiment/ALICE Collaboration.” http://aliceinfo.cern.ch/Public/Welcome.html

Grupo SCimago. 2012a. SCimago Country Rank. http://www.scimagojr.com/countryrank.php area $=0 \&$ category $=0 \&$ region $=$ Latin + America $\& y e a r=2012 \&$ or der $=$ it $\& \min =0 \&$ min_type $=$ it

Grupo SCimago. 2012b. SCimago Institutions Rankings. https://www.upjs.sk/public/ media/7379/sir-2012-world-report.pdf

Hung-Llamos, Blanca Rosa, Ricardo Arencibia-Jorge y Juan Antonio Araújo-Ruiz. 2008. "Identificación de frentes de investigación sobre esteroides en la producción científica cubana en Scopus 1996-2006.” ACIMED, 17 (3): 1-25. http://scielo.sld.cu/pdf/aci/v17n3/aci04308.pdf

ONEI (Oficina Nacional de Estadística e Información). 2012. Anuario Estadístico de Cuba 2012. http://www.one.cu/aec2012/esp/20080618_tabla_cuadro.htm

Rodríguez-Sánchez, Yaniris. 2010. "Metodología bibliométrica para la evaluación de la actividad científica." Tesis de doctorado, Instituto Superior de Tecnologías y Ciencias Aplicadas. http://catedragc.mes.edu.cu/download/Tesis\%20de\%20 Doctorado/Ingeniera\%20Industrial\%20-\%20Nacionales/Yaniris\%20Rodr\% C3\%ADguez\%20S\%C3\%A1nchezTESIS.pdf

Sancho, Rosa. 2003. "Directrices de la OCDE para la obtención de indicadores de Ciencia y tecnología.” Ministerio de Ciencia y Tecnología. España. http://micitt. go.cr/encuesta/docs/docs_tecnicos/ocde_directrices_para_indicadores_ciencia_y_tecnologia.pdf

Soriano-Torres, Michel, y Ricardo Arencibia-Jorge. 2010. "Estudio bibliométrico de la citogenética humana en el período 1999-2008." ACIMED 21 (4): 403-412. http://scielo.sld.cu/pdf/aci/v21n4/aci04410.pdf

UCI (Universidad de Ciencias Informáticas). 2002. Historia de la Universidad. https://www.uci.cu/?q=historia

WHO (World Health Organization). 2012. Levels and Trends in child mortality. http://www.who.int/maternal_child_adolescent/documents/levels_trends_child_ mortality_2012.pdf?ua=1

Para citar este texto:

Herrera-Vallejera, Darlenis, Rubén Sanchéz Perdomo, Marinelsy Rosario Sierra y Yaniris Rodríguez Sánchez. 2017. "Estudio cienciométrico de la actividad científica de Cuba en las Ciencias Naturales e Ingeniería y Matemática-Ciencias de la Computación”. Investigación Bibliotecológica: Archivonomía, Bibliotecología e Información 72 (31): 113-137. http://dx.doi.org/10.22201/iibi.0187358xp.2017.72.57826 